\title{
Frosted Branch Angiitis (FBA): A case report
}

\begin{abstract}
Saki $\mathrm{SY}^{1}$, Alam $\mathrm{M}^{2}$, Kadir $\mathrm{SMU}^{3}$

The purpose of the study is to describe a rare case report for raising the awareness of existence of this rare ocular disease and also review the characteristics of this disease.

A 35-year-old man presented with acute vision loss in both eye after high grade fever. On fundoscopic examination flower petal pattern blood vessel sheathing in temporal quadrant of both eyes was seen. A provisional diagnosis of frosted branch angiitis was considered. The patient responds promptly with Intravenous and oral corticosteroid. A total of 58 cases have been reported in worldwide literature. The disease is likely to represent a common immune pathway in response to multiple infective agents.
\end{abstract}

Key words: Retinal angitis, Vision loss.

CBMJ 2020 July: vol. 09 no. 02 P: $42-44$

\section{Introduction}

Frosted branch retinal angiitis (FBA) is a rare typically bilateral diffuse retinal periphlebitis. It describes a characteristics fundus picture, usually bilateral which is either primary or secondary due to cytomegalovirus retinitis, SLE, lymphoma. Leukemia. ${ }^{2}$ FBA typically affects children and young adult. It is a rare case, being described in only 58 cases in the literature most from Japan, but also some in North America, Turkey and India.

In 1976, Frosted branch angiitis was first described by Ito $Y$ et al. in a six-year Japanese boy presented with pan-uveitis and widespread retinal vasculitis. ${ }^{7}$ This entity is an acute pan-uveitis with severe vasculitis affecting the whole retina. Since veins are more involved than arteries, it is also called diffuse acute retinal periphlebitis. The florid translucent perivascular exudate was introduced the descriptive term 'frosted branch angiitis'. ${ }^{8}$

\section{Case Presentation:}

A 35 years old man presented with complaints of fever for 7 days \& unconscious for 1 day. His fever was high grade, intermittent, did not relieve by taking medication. Fever was gradually increasing in nature \& he started irrelevant talking 7 days after fever. Then he got admitted in a high dependency unit in a hospital, after 2 days his condition was improved. Then he noticed dimness of vision (B/E). On clinical examination his best corrected visual acuity $\mathrm{PL}$ in right eye \& $\mathrm{HM}$ in left eye. Ocular motility was full in all gazes, anterior segment of both eyes was normal. Posterior segment showed: blood vessel sheathing in temporal quadrant $(\mathrm{B} / \mathrm{E})$ which is flower petal pattern. There was dot, blot hemorrhage along blood vessel in both eye, optic disc size, shape \& foveal reflex were normal. His hemoglobin count was; 12.5 $\mathrm{g} / \mathrm{dl}$, neutrophil $79 \%$, lymphocyte $14 \%$, RBC, MCV, MCHC, Serum. Electrolyte count were within normal limit. Blood culture showed no organism. CSF study showed increased lymphocyte count (79\%)

1. Dr. Sadia Yeasmin Saki, Junior Consultant, Sheikh Fazilatunnessa Mujib Eye Hospital \& Training Institute, Gopalganj, Dhaka

2. Dr. Mezbahul Alam, Associate Professor, Department of Vitreo Retina, National Institute of Ophthalmology \& Hospital

3. Dr. Syeed Mehbub UI Kadir, Assistant Professor, Sheikh Fazilatunnessa Mujib Eye Hospital \& Training Institute, Gopalganj, Dhaka

Address of correspondence:

Email:sadia.y.saki@gmail.com 


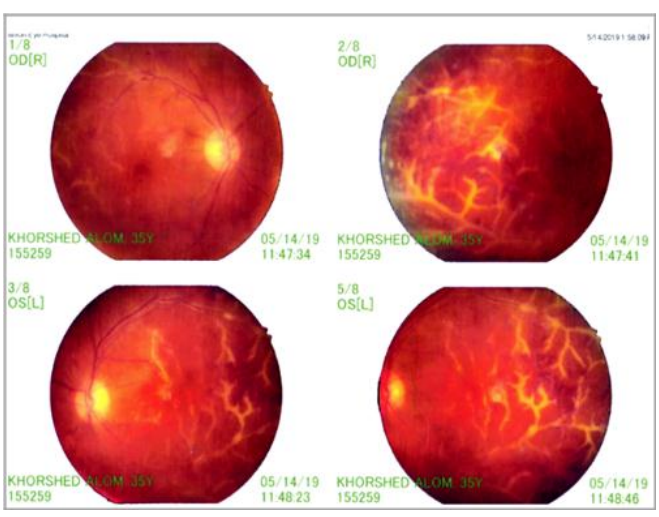

Management protocol:

Patient was diagnosed as a case of frosted branch angiitis due to cytomegalovirus. He was given I/V methylprednisolone for 3 days, then followed by oral steroid in a tapering dose, with oral proton pump inhibitor and oral calcium. After 2 week his vision in right eye was $6 / 18$ \& left eye was $6 / 24$. He was then advised for slow tapering dose of oral prednisolone.

\section{Discussion:}

The cause of FBA is idiopathic and may initiate an immune complex reaction.,10 FBA is classified the disease into 3 subgroups: idiopathic, associated with hematologic malignancies like leukemia and lymphoma, and those caused by viral infection or autoimmune disease. ${ }^{11}$ FBA is an uncommon inflammatory disorder principally involving posterior segment. It is commonly bilateral (75\%) disease and presented by acute/subacute visual loss, floaters or photopsiae. ${ }^{10}$ The common signs are severe vascular sheathing and retinal oedema, vitritis, iridocyclitis, and prompt response to corticosteroid but there is no clear guideline on treatment modality. ${ }^{10,12}$ It is important to exclude other causes of retinal vasculitis, especially for viral retinitis, sarcoidosis, multiple sclerosis, toxoplasmosis, syphilis, and Behcet's disease. Infiltrative lesions such as lymphoma and leukaemia may occasionally mimic FBA. ${ }^{13,14}$ The complication of frosted branch angiitis are neovascular glaucoma, macular scarring, retinal detachment, and neovascularization. ${ }^{15}$ Nevertheless, despite the severe retinal appearance, the prognosis is usually good, with rapid recovering of visual acuity after prompt steroid treatment. Visual field and electrophysiological tests return completely normal after one or two months from onset.

\section{Conclusion:}

This case report therefore emphasizes the need of considering a rare disease which with prompt corticosteroid treatment carries a good visual prognosis.

\section{Consent:}

Written informed consent was obtained from the patient for publication of this case report, color fundus photographs, and any accompanying image.

\section{References:}

1. Wood EH, Wong RW. Bilateral frosted branch angiitis as the presenting sign of antiphospholipid antibody syndrome. Journal of ophthalmic inflammation and infection. 2016 Dec; 6(1):20

2. Miserocchi E. Frosted Branch Angiitis. url:https://www.uveitis.org/wpcontent/uploads /2017/05/frosted_branch_angiitis.pdf

3. Watanabe YO, Takeda NO, Adachi-Usami EM. A case of frosted branch angiitis. British journal of ophthalmology. 1987 Jul 1; 71(7):553-8.

4. Fine HF, Smith JA, Murante BL, Nussenblatt $R B$, Robinson MR. Frosted branch angiitis in a child with HIV infection. American journal of ophthalmology. 2001 Mar 1; 131(3):394-6

5. Geier SA, Nasemann J, Klauss V, Kronawitter U, Goebel FD. Frosted branch angiitis in a patient with the acquired immune deficiency syndrome. American journal of ophthalmology. 1992 Feb 1; 113(2):203-5.

6. Walker $S$, Iguchi $A$, Jones NP. Frosted branch angiitis: a review. Eye. 2004 May; 18(5):527.

7. Ito $Y$, Nakano M, Kyu N, Takeuchi M. Frosted branchangiitis in a child.Rinsho Ganka (Jpn J Clin Ophthalmol)1976;30: 797-803 
8. Huerva V, Puig $T$, Sanchez MC, Jurjo $C$, Asenjo J. A new case of acute idiopathic frosted branch angiitis in Europe. Eyr $J$ Ophthalmol2002;12: 127-130.

9. Mizukami Y, Yagasaki T, Shimazu K, Yagasaki K. A 5-year-old girl with frosted branch angiitis of theretina.Nihon Ganka Kiyo (Folia Ophthalmol Jpn)1990;41:2080-2087.

10. Okinami S, Nakamatsu T, Saito I, Oohira A, Yoshida M, Yamakawa R, Ishikawa M, Miyara $N$. Four cases of frosted retinal angiitis. Nihon Ganka Kiyo (Folia Ophthalmol Jpn) 1994;45: 314-318.

11. Kanai K, Nomura T, Fukuda S, Takahashi Y, Kogure M. An adult case of frosted branch angiitis. Ganka Rinsho Iho (Jpn Rev Clin Ophthalmol)1989;83: 1681-1683.

12. Kawaguchi S, Yasuda F, Kawamura T, Hara J. A case offrosted branch angiitis. Nihon Ganka Kiyo (Folia Ophthalmol Jpn)1996;47: 1421-1425.

13. Biswas J, Raizada S, Gopal L, Kumarasamy $N$, Solomon S. Bilateral frosted branch angiitis and cytomegalovirus retinitis in acquired immunodeficiency syndrome. Indian JOphthalmol1999;47: 195-197.

14. Marko-michelakis NN, Barampouti F, Zafirakis $P$, Chalkiadakis I, Kouris T, Ekonomopoulos $N$. Retinal vasculitis with a frosted branch angiitis-like response due toherpes simplex virus type 2. Retina 1999;19(5): 455-457.

15. Kleiner RC, Kaplan HJ, Shakin JL, et al. Acute frosted retinal periphlebitis. Am J Ophthalmol 1988; 106: 27-34. 